\title{
Câncer de pele: caracterização do perfil e avaliação da proteção solar dos pacientes atendidos em serviço universitário
}

\section{Skin cancer: profile characterization and assessment to sun protection in patients of a college service}

\author{
Carla Andréa Avelar Pires ${ }^{1}$, Aglayn Pimentel Fayal ${ }^{2}$, Renata Henriques Cavalcante ${ }^{3}$, Syenne Pimentel Fayal ${ }^{4}$, Nicole Salomão \\ Lopes $^{4}$, Franklin Pimentel Fayal ${ }^{5}$, Maria Amélia Lopes dos Santos ${ }^{1}$ \\ 1. Docente do curso de Medicina da Universidade do Estado do Pará e Universidade Federal do Pará, Belém, PA, Brasil. 2. Residente de Saúde da Família da \\ Universidade do Estado do Pará, Belém, PA, Brasil. 3. Residente de Dermatologia pela Universidade Federal do Pará, Belém, PA, Brasil. 4. Discente do curso \\ de medicina pela Universidade do Estado do Pará, Belém, PA, Brasil. 5. Discente do curso de medicina pela Universidade Federal do Pará, Belém, PA, Brasil.
}

\section{Resumo}

Introdução: 0 câncer de pele é a neoplasia de maior incidência no Brasil. Vários fatores têm sido atribuídos como risco para seu desenvolvimento, como: cor da pele, horário e tempo de exposição ao sol, residência em um país tropical e uso de imunossupressão crônica. Objetivo: descrever os casos de câncer de pele, bem como o hábito de proteção solar na prevenção de lesões causadas por radiação ultravioleta. Métodos: estudo transversal, individualizado e observacional com 50 pacientes, com diagnóstico de câncer de pele, acompanhados no Ambulatório de Dermatologia da Universidade do Estado do Pará, na cidade de Belém (PA), no período de janeiro de 2013 a outubro de 2016. A pesquisa foi feita por meio de um questionário padronizado autoaplicativo. Resultados: Observou-se o maior acometimento de câncer de pele em pacientes com idade > 50 anos (90\%), brancos (68\%), olhos castanhos (68\%), cabelos castanhos (78\%), que não faziam uso de filtro solar (86\%); com diagnóstico de Carcinoma Basocelular (94\%), tendo a face como a área corporal mais acometida (52\%). Conclusões: 0 carcinoma Basocelular com lesões de mais de um ano de surgimento foi o câncer de pele predominante nesta população em que a maioria não apresentava o hábito do uso de filtro solar.

Palavras-chave: Câncer de Pele. Perfil. Proteção solar.

\begin{abstract}
Introduction: Skin cancer is the cancer with the highest incidence in Brazil. Several factors have been assigned as a risk for its development, such as skin color, exposure time to the sun, living in a tropical country, making use of chronic immunosuppression. Objective: describe the cases of skin cancer and sun protection habits in preventing lesions caused by ultraviolet radiation. Methods: A cross-sectional, individuated and observational study with 50 patients, with the diagnosis of skin cancer accompanied at the dermatology clinic in the Pará State University, in Belém (PA), in the period of October 2016. Results: the greatest involvement of skin cancer was observed in patients with the following characteristics: age > 50 years (90\%), White (68\%), Brown eyes (68\%), Brown hair (78\%), did not use sunscreen (86\%); as well as the predominance of Basal Cell Carcinoma in patients (94\%), having the face as the most affected body area $(52 \%$. Conclusion: Basal Cell Carcinoma with lesions more than one year old was the predominant skin cancer in the population, in which the majority did not have the habit of using sunscreen.
\end{abstract}

Key words: Skin cancer. Profile. Solar protection.

INTRODUÇÃO

O câncer de pele é a neoplasia de maior incidência no Brasil. ${ }^{1}$ Vários fatores têm sido atribuídos ao risco para o desenvolvimento dessas neoplasias, como: cor da pele, horário e tempo de exposição ao sol, residência em país tropical e uso de imunossupressão crônica. ${ }^{2}$

O câncer de pele é mais comum em pessoas com mais de 40 anos, sendo relativamente raro em crianças e negros. Pessoas de pele clara, sensíveis à ação dos raios solares ou com doenças cutâneas prévias são as principais vítimas. ${ }^{2}$

Segundo Mortagner e Costa (2009) ${ }^{3}$, a exposição solar, devido à radiação ultravioleta, intensifica o fotoenvelhecimento e aumenta a chance de câncer de pele.
O câncer da pele é comumente dividido em não melanoma (Carcinoma Basocelular ou Carcinoma Epidermoide) e melanoma. $^{2}$

O melanoma é o mais agressivo e é classificado em grupos clínico-histológicos: melanoma em lentigo maligno, melanoma disseminativo superficial, melanoma nodular, e melanoma acral lentiginoso. 40 tipo não melanoma é o câncer mais frequente no Brasil .

O Carcinoma Basocelular (CBC) constitui o tipo mais comum de carcinoma da pele. Ocorre, predominantemente, em áreas expostas ao sol, principalmente, nas regiões da cabeça e do pescoço ( $80 \%$ dos casos), seguido do tronco $(15 \%)$, das mãos 
e pernas ${ }^{5}$. É mais frequente em homens brancos com idade superior a 40 anos $^{6}$. Os CBCs são classificados, de acordo com características clínicas e anatomopatológicas em: nodular, ulcerado, superficial, esclerodermiforme e metatípico ${ }^{7}$. A terapêutica de escolha permanece a exérese cirúrgica ${ }^{8}$.

O Carcinoma Espinocelular (CEC) é resultado da radiação solar cumulativa durante toda a vida9 e representa $20 \%$ das neoplasias malignas cutâneas, podendo surgir a partir de lesões não invasivas como Ceratoses Actínicas (CA), Queilites Actínicas, Leucoplasias Orais e Radiodermites Crônicas ${ }^{10}$.

O diagnóstico deve ser baseado na história e nas manifestações clínicas do paciente, e, principalmente, no exame histopatológico da lesão? .

Denominam-se dermatoses pré-cancerosas aquelas que podem evoluir para um verdadeiro câncer cutâneo. Entre as dermatoses pré-cancerosas mais importantes, estão: Ceratose Actínica, Doença de Bowen, Nevos, Queilites e Úlcera de Marjolin. ${ }^{12}$

A Ceratose Actínica, solar ou, impropriamente, senil, é a lesão pré-maligna mais frequente, que ocorre em áreas expostas à luz solar, em pessoas idosas ou adultas de meia idade e em pele clara. $^{13}$

A Doença de Bowen (DB) é o verdadeiro Carcinoma Espinocelular in situ. ${ }^{12} \mathrm{~A}$ presença do nevo atípico é um fator importante associado ao risco aumentado de desenvolvimento do melanoma cutâneo. ${ }^{14}$

Queilite Actínica é uma lesão benigna caracterizada por eritema do lábio inferior. Incide principalmente em indivíduos acima dos 45 anos, tendo preferência pelo sexo masculino. ${ }^{15}$

Objetiva-se, com a presente pesquisa, caracterizar o perfil dos pacientes com câncer de pele, bem como abordar o hábito de proteção solar nos pacientes atendidos pelo Ambulatório de Dermatologia da Universidade do Estado do Pará no período de janeiro de 2013 a janeiro de 2016.

\section{METODOLOGIA}

É um estudo transversal, individual e observacional com 50 pacientes com diagnóstico de câncer de pele, atendidos no Ambulatório de Dermatologia da Universidade do Estado do Pará na cidade de Belém (PA), no período de janeiro de 2013 a janeiro de 2016.

A população da amostra foi constituída de pacientes acima de 18 anos, de ambos os sexos, diagnosticados com câncer de pele, comprovados por meio do exame histopatológico, realizado no serviço. Eles eram atendidos no Serviço de Dermatologia da Universidade do Estado do Pará, no período de outubro de 2016, tendo o diagnóstico sido feito de janeiro de 2013 até janeiro de 2016. O tamanho da amostra do estudo foi justificada pela quantidade de prontuários disponíveis com informações completas, que viabializaram o preenchimento satisfatório do protocolo.

A pesquisa foi feita por meio da aplicação de questionário autoaplicativo, composto por 29 itens, elaborado pelos pesquisadores e aplicados por duas acadêmicas do $6^{\circ}$ ano de Medicina. Coletaram-se dados sobre identificação pessoal (sexo, idade, procedência e profissão), características fenotípicas (cor da pele, cor dos cabelos e dos olhos), hábitos de exposição solar (hábito de se expor ao sol para bronzeamento natural, tempo de exposição solar diário, exposição solar ocupacional, realização de bronzeamento artificial), medidas preventivas à fotoexposição (utilização e frequência de uso de fotoprotetor ou outros métodos), presença de lesões de pele preexistentes (câncer de pele anterior, lesão pré-maligna, fatores irritativos crônicos, HPV, úlceras angiodérmicas e cicatriz de queimadura), tempo de procura por atendimento médico após o aparecimento da lesão e tempo de diagnóstico. Ao todo, 50 questionários foram respondidos.

Foram aplicados métodos estatísticos descritivos e inferenciais. A estatística descritiva foi aplicada de três maneiras: tabelas, gráficos e medidas estatísticas de posição, tendo como principal objetivo descrever e reduzir os dados para que se tenha uma caracterização e uma melhor visualização desses dados de forma clara e objetiva. A inferência estatística foi implementada por meio de teste de hipótese: Teste G, que comparou as proporções dentro da amostra e entre duas amostras independentes conforme o caso analisado. Foi previamente estabelecido o nível de significância alfa $=0.05$ para a rejeição da hipótese de nulidade e uma confiabilidade de $95 \%$. Todo o processamento estatístico foi realizado sob o suporte computacional do software, bioestat 5.3 e Microsoft Office Excel 2010.

O estudo foi aprovado pelo Comitê de Ética da Uepa, CAAE: 53249015.2.0000.5174. Os objetivos da pesquisa foram explicados durante a aplicação dos questionários, e os pacientes foram assegurados do total anonimato, aceitando participar da pesquisa por meio da assinatura do TCLE.

\section{RESULTADOS}

CNa análise do perfil sociodemográfico dos pacientes diagnosticados com câncer de pele, entre janeiro de 2013 a janeiro de 2016, acompanhados pelo serviço de dermatologia, observou-se que metade dos paciente era do sexo masculino, e a maioria tinha idade entre 50 a 80 anos. Em relação à profissão, nem todos os indivíduos trabalhavam a céu aberto, e o emprego mais citado foi o de pedreiro. 
Tabela 1. Características sociodemográficas dos pacientes diagnosticados com câncer de pele

\begin{tabular}{lrr}
\hline Variável & $\mathbf{N}(\mathbf{5 0})$ & $\%$ \\
\hline Gênero & 25 & 50,0 \\
Feminino & 25 & 50,0 \\
Masculino & & \\
Idade & 5 & 10,0 \\
$\geq 35$ e <50 anos & 20 & 40,0 \\
$\geq 50$ e $<65$ anos & 23 & 46,0 \\
$\geq 65$ e <80 anos & 2 & 4,0 \\
$\geq 80$ anos & & \\
Profissão & 3 & 6,0 \\
Aposentado & 14 & 14,0 \\
Doméstica & 2 & 4,0 \\
Lavadeira & 7 & 14,0 \\
Lavrador & 3 & 26,0 \\
Motorista & 8 & \\
Pedreiro & 13 & \\
Outros & & \\
\hline
\end{tabular}

Fonte: protocolo de pesquisa

Em um total de 50 casos, foi avaliado o tempo de diagnóstico de câncer de pele, período que variou entre menos de um mês e mais de um ano, sendo predominante o tempo inferior a um mês, apresentado por 18 pacientes (36\%), tal como mostra a tabela 2.

Tabela 2. Tempo de diagnóstico dos pacientes diagnosticados com câncer de pele.

\begin{tabular}{lrr}
\hline Tempo de diagnóstico & Casos & $\%$ \\
\hline$<1$ Mês & 18 & 36,0 \\
$>1$ e $<3$ Meses & 14 & 28,0 \\
$>3$ e <6 Meses & 12 & 24,0 \\
$>1$ Ano & 6 & 12,0 \\
\hline Total & 50 & 100,0 \\
\hline
\end{tabular}

Fonte: protocolo de pesquisa

De acordo com a tabela 3, a qual mostra a correlação encontrada entre cor da pele e diagnóstico de câncer de pele, o Carcinoma Basocelular predominou em pacientes de cor branca, e o Carcinoma Espinocelular acometeu, majoritariamente, os de cor parda. Os pacientes de cor negra não apresentaram Carcinoma Espinocelular. Do grupo de pacientes diagnosticados com Carcinoma Basocelular, os negros compunham $9 \%$.

Na tabela 4, percebe-se, pela relação entre o uso de filtro solar com o tipo histológico de câncer de pele, que a maioria dos indivíduos que tiveram diagnóstico de Carcinoma Basocelular e todos com diagnóstico de Carcinoma Espinocelular não faziam uso de filtro solar.

Tabela 3. Correlação do diagnóstico com a cor da pele dos pacientes diagnosticados com câncer de pele

\begin{tabular}{lrrrrr}
\hline Pele & $\begin{array}{r}\text { Carcinoma } \\
\text { Basocelular }\end{array}$ & $\%$ & $\begin{array}{r}\text { Carcinoma } \\
\text { Espinocelular }\end{array}$ & $\%$ & Total \\
\hline Brancos & 33 & 70,0 & 1 & 33,0 & 34 \\
Pardos & 10 & 21,0 & 2 & 67,0 & 12 \\
Negro & 4 & 9,0 & 0 & 0,0 & 4 \\
\hline Total & 47 & 100,0 & 3 & 100,0 & 50 \\
\hline Fonte: protocolo de pesquisa. & & & $\mathrm{P}<0,05$ &
\end{tabular}

Tabela 4. Relação entre uso de filtro solar e tipo histológico de câncer de pele

\begin{tabular}{lrrrrrr}
\hline $\begin{array}{l}\text { Uso de } \\
\text { filtro solar }\end{array}$ & $\begin{array}{r}\text { Carcinoma } \\
\text { Basocelular }\end{array}$ & $\begin{array}{l}\text { Carcinom a } \% \\
\text { Espinocelular }\end{array}$ & Total \\
\hline Sim & 7 & 15,0 & 0 & 0,0 & 7 \\
Não & 40 & 85,0 & 3 & 100,0 & 43 \\
\hline Total & 47 & 100,0 & 3 & 100,0 & 50 \\
\hline Fonte: protocolo de pesquisa. & & & \multicolumn{2}{c}{$\mathrm{P}<0,05$}
\end{tabular}

Também foi estabelecida a relação entre tipo histológico de câncer de pele e cor dos olhos e dos cabelos dos paciente diagnosticados, a qual revelou que a maioria dos indivíduos diagnosticados com Carcinoma Basocelular e todos aqueles com Carcinoma Espinocelular apresentam olhos castanhos e cabelos castanho-escuros, como evidencia a tabela 5.

Também foi analisado o tempo diário de exposição solar do paciente, o que revelou que a maioria (38\%) dos indivíduos ficava exposta durante um período de 1 a 3 horas, $26 \%$ de 4 a 6 horas, $30 \%$ de 7 a 10 horas e $6 \%$ acima de 10 horas.

\section{DISCUSSÃO}

O câncer de pele foi encontrado em ambos os sexos com percentual iguais. Nigro et al. (2015)' 6 encontraram pouca diferença também na ocorrência quanto ao sexo, em que de 1.150 pacientes, $51,2 \%$ eram mulheres. A discrepância é citada em trabalhos como os de Souza et al (2011)'7 e o INCA (2014)², em que comentam sobre a ocorrência de uma mudança no padrão de acometimento do câncer, aumentando o número de casos em mulheres. Também, segundo esses autores, este fato pode ser explicado pela cultura da pele bronzeada, que é mais incidente no sexo feminino.

Quanto à idade, o câncer de pele preponderou entre 5080 anos, como já esperado, visto que o dano causado pelos raios ultravioletas é cumulativo, levanto a lesões fotoquímicas progressivas, dado também encontrado por Simoneti et al. (2016) ${ }^{1} 8$ na cidade de São Paulo, onde a idade destacada foi igual ou superior a 60 anos, em 71,6\% da amostra. 
Tabela 5. Relação entre cor do cabelo, cor dos olhos e tipo histológico de câncer dos pacientes diagnosticados com câncer de pele

\begin{tabular}{|c|c|c|c|c|c|c|c|c|c|c|c|}
\hline $\begin{array}{l}\text { Cor do } \\
\text { cabelo }\end{array}$ & $\begin{array}{l}\text { Carcinoma } \\
\text { Basocelular }\end{array}$ & $\%$ & $\begin{array}{r}\text { Carcinoma } \\
\text { Espinocelular }\end{array}$ & $\%$ & Total & $\begin{array}{r}\text { Cor dos } \\
\text { olhos }\end{array}$ & $\begin{array}{l}\text { Carcinoma } \\
\text { Basocelular }\end{array}$ & $\%$ & $\begin{array}{r}\text { Carcinoma } \\
\text { Espinocelular }\end{array}$ & $\%$ & Total \\
\hline $\begin{array}{l}\text { Castanho } \\
\text { escuro }\end{array}$ & 21 & 45,0 & 3 & 100,0 & 24 & $\begin{array}{l}\text { Castanho } \\
\text { escuro }\end{array}$ & 18 & 38,0 & 0 & 33,0 & 2 \\
\hline $\begin{array}{l}\text { Castanho } \\
\text { claro }\end{array}$ & 15 & 32,0 & 0 & 0,0 & 15 & $\begin{array}{l}\text { Castanho } \\
\text { claro }\end{array}$ & 13 & 28,0 & 2 & 67,0 & 17 \\
\hline Loiro & 6 & 13,0 & 0 & 0,0 & 6 & Verde & 9 & 19,0 & 0 & 0,0 & 9 \\
\hline \multirow[t]{2}{*}{ Preto } & 5 & 10,0 & 0 & 0.0 & 5 & Azul & 2 & 13,0 & 0 & 0,0 & 2 \\
\hline & & & & & & Preto & 1 & 2,0 & 0 & 0,0 & 1 \\
\hline Total & 47 & 100,0 & 3 & 100,0 & 50 & Total & 47 & 100,0 & 3 & 100,0 & 50 \\
\hline
\end{tabular}

Entre as mulheres, a profissão mais comum era de doméstica. Quanto aos homens, foi a de pedreiro. Ambas apresentam exposição solar ocupacional, somado ao fato de Belém ser uma região com clima equatorial, onde a incidência de raios solares é alta. Estes resultados estão em consonância com Ferraz et al. $(2012)^{1} 9$, em que a maior parte dos pacientes com câncer de pele trabalhavam expostos ao sol.

Da amostra, quase a totalidade foi diagnosticada com Carcinoma Basocelular. Em concordância com os estudos de Simoneti et al. (2016) 8 , que foram realizados em São Paulo, nos quais $69,2 \%$ dos indivíduos tinham Carcinoma Basocelular. Quase a totalidade dos estudos feitos no Brasil sobre o tema obteve dominância de Carcinoma Basocelular, o que reflete o fato de esta ser a principal causa de câncer no Brasil (INCA, 2014)².

Dos diagnosticados com Carcinoma Espinocelular, a maioria possuía cor parda, olhos castanho-claros, cabelo castanhoescuro e mais de 60 anos. Todos os três casos encontrados eram em homens que não utilizavam filtro solar. Para Barrela et al. (2013) ${ }^{10}$, que pesquisaram apenas sobre Carcinoma Espinocelular, constatou-se preponderância em mulheres $(55,4 \%)$ maiores de 60 anos $(89,3 \%)$. Para eles, contudo, não houve significância estatística entre a cor de cabelo e olhos e a neoplasia. A explicação para tal resultado reside no fato de estudos epidemiológicos indicarem que a exposição solar cumulativa (principalmente radiação UVB) é a mais importante causa ambiental do carcinoma de células escamosas cutâneo; portanto, quanto maior a idade, maior a probabilidade de desenvolvê-lo. (MARTINEZ e col, 2006) ${ }^{20}$.

Em concordância com grande parte dos trabalhos publicados sobre o tema, o perfil epidemiológico prevalente foi de pessoas com pele branca, idosas, olhos castanho-escuros e cabelos castanho-escuros. Houve domínio destas características também para Silva et al $(2016)^{21}$ e Nigro et al. (2015) 6 . Peles mais claras são, sabidamente, mais propensas ao desenvolvimento de tumores neoplásicos epiteliais. Entretanto, segundo Araújo et al $(2015)^{22}$, o fato de não brancos também possuírem o câncer aponta para a importância dos outros fatores de risco implicados no aumento da incidência das neoplasias de pele, como: exposição excessiva à radiação solar, idade avançada, hábito de fumar, abuso de álcool, entre outros.
As áreas mais expostas ao sol são as de maior incidência da lesão. A face foi onde se diagnosticaram mais lesões, principalmente no nariz, concordando com a literatura. Silveira $(2014)^{23}$ referiu, em sua investigação, os locais mais comuns de lesão e constatou que foram: face (38\%), braços (38\%), pernas (33\%). Isso demonstra a relevância do papel da exposição ao sol como fator de risco e consequente aumento do desenvolvimento de neoplasias cutâneas nessas regiões.

A lesão existe há mais de um ano em praticamente todos os participantes do estudo. Todavia, na maioria dos casos, a procura por atendimento médico e posterior diagnóstico ocorreu há menos de um mês. O intervalo predominante do tempo decorrido entre surgimento da lesão e estabelecimento do diagnóstico foi de aproximadamente 11 meses. Pode-se supor que parte da população não dá a devida importância ao surgimento de lesões na pele, principalmente quando são pouco sintomáticas, e, quando procuram atendimento médico, também esbarram na dificuldade da aquisição de atendimento e acompanhamento no sistema de saúde público. Tendo em vista que as neoplasias cutâneas são advindas de lesões progressivas e podem ser prevenidas, pode-se, então, afirmar que quanto maior for o tempo entre o aparecimento da lesão e o diagnóstico, mais tardiamente se dará o tratamento e maiores serão as repercussões na qualidade de vida dos pacientes. Aquino et al. $(2016)^{2} 4$, em suas variáveis encontradas, concordam com os dados aqui descritos.

Dos pacientes encontrados no presente estudo, a grande maioria se expunha ao sol de forma ocupacional, discordando do estudo de Castilho et al. (2010)' , realizado em Brasília com 368 pacientes, o qual constatou que a maior parte da amostra se expunha ao sol por lazer (66,9\%). Apesar de as duas cidades terem alto índice de insolação (ANEEL, 2016) 5 , tal divergência pode ser explicada pela diferença etária da população das pesquisas, Castilho et al. (2010) ${ }^{1}$ tiveram uma amostra com média de idade mais jovem ( $22,1 \pm 5,2$ anos); é provável que esse comportamento esteja relacionado a atividades sociais, prática de esportes ao ar livre e intenção de aproveitar mais o dia.

O tipo de proteção mais utilizado no dia a dia dos entrevistados foi chapéu ou boné. Percebeu-se que menos de um sexto 
utiliza filtro solar, entre os quais, quase a totalidade não o usava diariamente. Ferraz et al. (2012) 19 identificaram a predominância do uso de proteções simples como chapéus e camisas de manga longa (60\%) e perceberam, ademais, que apenas $31 \%$ utilizavam filtro solar, alertando para o fato de que ainda se tem uma baixa adesão ao uso de filtro solar, corroborando o alto nível de atendimento ambulatorial de lesões cutâneas causadas pelo fotodano.

Uma pequena parcela dos pacientes alegou fazer uso de filtro solar. Em discrepância a este estudo, Castilho et al. (2010) ${ }^{1}$ concluíram que mais da metade de sua amostra, 83,9\%, utilizava filtro solar. Nota-se, portanto, que pode haver, por parte da população, falta de informação sobre câncer de pele, como o uso de filtro solar pode preveni-la, bem como seu modo de uso; porém, o fato do alto custo do produto também pode influenciar na não adesão a ele.

Infelizmente, um número significativo de entrevistados afirma possuir hábito de bronzeamento natural (22\%). O estudo de Castilho et al. (2010) ${ }^{1}$, realizado em 368 pacientes, em Brasília, detectou bronzeamento natural em $12 \%$ de seus entrevistados. Destes, a maioria o fazia pela manhã e à tarde, em consonância com o estudo aqui discutido.

Mesmo com esforços de campanhas educativas alertando sobre os efeitos nocivos da fotoexposição em horários de risco, a exposição solar diária apareceu com prevalência em períodos $>7$ h por dia. Pode-se justificar tal período pelo perfil epidemiológico de profissões dos entrevistados, visto que a maioria tem exposição ocupacional. Ferraz et al. (2012) 19 observaram como tendência de seus pacientes trabalhar mais de 8 horas diárias expostos ao sol. Tendo em vista esses dados, nota-se a necessidade do uso de medidas protetoras por trabalhadores que realizam atividades ao ar livre, como o uso de protetor solar, camisas e chapéus.

A história familiar estava presente em uma pequena parte da amostra, devido a um viés de memória, visto que muitos não lembravam ou não tinham contato com a família. O mesmo obstáculo foi enfrentado por Castilho et al (2010) ${ }^{1}$, observando também uma minoria de relato de câncer de pele na família e alegando tal viés dos entrevistados.

Ao observar os resultados, pode-se visualizar a necessidade de melhoria no acesso da população ao atendimento, quanto à obtenção de informações sobre esta doença, a qual ainda se apresenta como o principal tipo de câncer no mundo, no intuito de aumentar as medidas preventivas tomadas pelos indivíduos, como o uso de filtro solar e barreiras físicas, bem como mais atenção às lesões de pele.

\section{CONCLUSÃO}

A maior ocorrência de diagnóstico é o Carcinoma Basocelular. Quanto à localização das lesões, a face foi a mais acometida, principalmente o nariz, seguida de membros superiores e dorso. O perfil epidemiológico caracteriza-se por: maiores de 50 anos, brancos, olhos e cabelos castanho-escuros, sem prevalência entre os sexos. Doméstica e pedreiro foram as profissões que preponderaram. Os não usuários de filtro solar são sobressalentes. Predominam lesões existentes há mais de um ano; no entanto, a procura por atendimento médico e posterior diagnóstico ocorreu após meses a anos do aparecimento da lesão.

\section{REFERÊNCIAS}

1. Castilho IG, Sousa, MAA, Leite, RMS. Fotoexposição e fatores de risco para câncer da pele: uma avaliação de hábitos e conhecimentos entre estudantes Universitários. An Bras Dermatol. 2010 Mar-Abr; 85(2): 173-8. doi: http:// dx.doi.org/10.1590/S0365-05962010000200007

2. Instituto nacional de câncer. Pele não melanoma [internet]. Rio de Janeiro: INCA; 2014 [acesso 2015 Jul 10]. Disponível em: http://www2.inca.gov.br/wps/ wcm/connect/tiposdecancer/site/home/pele_nao_melanoma.

3. Montagner S, Costa, A. Bases biomoleculares do fotoenvelhecimento. An. Bras. Dermatol. 2009. 84 (3):91-8.

4. Souza SRP, Fischer, FM, Souza, JMP. Bronzeamento e risco de melanoma cutâneo: revisão da literatura. Rev Saúde Pública. 2004 Ago. 38.(4):588-98. doi: http://dx.doi.org/10.1590/S0034-89102004000400018.

5. Custódio G, Locks LH, Coan MF, Gonçalves CO, Trevisol DJ, Trevisol FS Epidemiologia dos carcinomas basocelulares em Tubarão, Santa Catarina (SC), Brasil, entre 1999 e 2008. An Bras Dermatol. 2010 Nov-Dez. 85(6): 815-26. doi: http://dx.doi.org/10.1590/S0365-05962010000600007.

6. Ferreira CB, Diniz LM, Souza JB. Múltiplos carcinomas basocelulares na região pubiana em uma paciente fototipo IV - Relato de caso. An Bras Dermatol. 2011. 86(3): 589-91. doi: http://dx.doi.org/10.1590/S0365-05962011000300030.

7. Santos El, Goulart EF, Barreto LM, Vâncio MS, Mota DB, Andrade VLFS, et al. Úlcera de Marjolin: evidências científicas e perspectivas atuais para a enfermagem. Revista Eletrônica Gestão \& Saúde.2015; 6(01):472-86.
8. Schimitt JV, Chimen VP, Marques MEA, Miot HA. Aumento da incidência de carcinoma basocelular em hospital universitário: 1999 a 2009. An Bras Dermatol. 2011; 86(2):375-7. doi: http://dx.doi.org/10.1590/S0365-05962011000200029.

9. Barella CS, Blanco LFO, Yamane A. Análise dos dados epidemiológicos dos laudos de carcinoma espinocelular. Rev Bras Clin Med. 2013 Jan-Mar; 11(1): 43-7.

10. Dornelas MT, Rodrigues MF, Machado DC, Gollner A.M, Ferreira A.P. Expressão de marcadores de proliferação celular e apoptose no carcinoma espinocelular de pele e ceratose actínica. An. Bras. Dermatol. 2009 Set-Out; 84(5): 469-75. doi: http://dx.doi.org/10.1590/S0365-05962009000500004.

11. Abreu, MAMM, Pimentel DRN, Silva, OMP, Blachman IT, Michalany NS. Hirata $\mathrm{CH}$, et al. Carcinoma espinocelular do lábio:avaliação de fatores prognósticos. Rev Bras Otorrinolaringol. 2004 Nov-Dez; 70(06):765-70. doi: http://dx.doi. org/10.1590/S0034-72992004000600010.

12. Azulay RD. Dermatologia. 5. ed. São Paulo: Guanabara Koogan; 2011. p. 590-4.

13. Poziomczykl CS, Kochell B, Dornelles MA, Dornelles SIT. Avaliação da dor em criocirurgia de ceratoses actínicas. An. Bras. Dermatol. 2011; 86(4): 654-8. doi: http://dx.doi.org/10.1590/S0365-05962011000400003.

14. Rezze GG, Leon A, Duprat J. Nevo displásico (nevo atípico). An. Bras. Dermatol. 2010 Nov-Dez; 85(6): 863-71. doi: http://dx.doi.org/10.1590/S036505962010000600013 
15. Corso FM, Wild C, Gouveia LO, Ribas MO. Queilite actínica: prevalência na ciência estomalogógica da PUCPR, Curitiba, Brasil. Clin. Pesq. Odontol. 2006 Abr-Jun; 02(04): 277-81.

16. Nigro MHMF. Estudo epidemiológico do carcinoma basocelular no período de 2010 a 2013 em um hospital de referência em dermatologia na cidade de Baurú, São Paulo. [monografia]. São Paulo (SP): Instituto Lauro de Souza Lima; 2013.

17. Souza CFD, Thomé EP, Manegotto PF, Schmiti JV, Shibue JRT, Tarlé JR. Topografia do carcinoma basocelular e suas correlações com o gênero, a idade e o padrão histológico: um estudo retrospectivo de 1.042 lesões. An Bras Dermatol. 2011 Mar-Abr; 86(2): 272-7. doi: http://dx.doi.org/10.1590/S036505962011000200010

18. Simoneti F, Cunha LO, Gomes CTV, Novo NF, Portella DL, Gonella HA. Perfi epidemiológico de pacientes com tumores cutâneos malignos atendidos em ambulatório de cirurgia plástica de serviço secundário no interior de São Paulo. Rev Fac Ciênc Méd Sorocaba. 2016; 18(2): 98-102. doi: http://dx.doi. org/10.5327/z1984-4840201624713.

19. Sousa TFS, Silva LS, Cunha CRM, Taminato RL. Avaliação dos hábitos relativos a fotoexposição e sensibilização quanto a fatores de risco para câncer de pele em trabalhadores rurais. Rev. Saúde.com. 2012; 98(7): 11-16.
20. Martinez MAR, Francisco G, Cabral LS, Ruiz IRG, Festa C Neto. Genética molecular aplicada ao câncer cutâneo não melanoma. An. Bras. Dermatol. 2006; 8(05): 405-19.

21. Silva AKD, Santos FG, Haeffner LSB, Budel F, Farenzena GJ, Bebe AAC. Câncer de pele: demanda de um serviço de dermatologia de um hospital terciário. Saúde (Santa Maria). 2012 Jul-Dez; 38(2):55--64. doi: http://dx.doi. org/10.5902/223658345660.

22. Bezerra CA, Maia, PCGGS, Thigueiro GPT, Sousa MNA. Conhecimentos, atitudes e práticas de agricultores sobre a prevenção do câncer de pele. Rev. Saúde Públ. 2015 Dez; 8(3): 8-18.

23. Silveira TLA. Associação entre lesões sugestivas de câncer de pele e exposição solar ocupacional em pescadoras artesanais de Saubara, Bahia, Brasil. [monografia]. Salvador (BA): Faculdade de Medicina da Bahia; 2014.

24. Aquino RCA, Rodigues M. Acesso e itinerário terapêutico dos pacientes com câncer: principais dificuldades enfrentadas para este percurso. Rev. Saúde. com.2016; 12(6): 488-96. doi: ttp://dx.doi.org/10.22481/rsc.v12i1.317.

25. energia solar [internet]. Rio de Janeiro: ANEEL; 2016 [acesso 2016 Set 8]. Disponível em: http://www2.aneel.gov.br/aplicacoes/atlas/energia_solar/3_2. htm.

\section{Como citar este artigo/How to cite this article:}

Pires CAA, Fayal AP, Cavalcante RH, Fayal SP, Lopes NS, Fayal FP, et al. Câncer de pele: caracterização do perfil e avaliação da proteção solar dos pacientes atendidos em serviço universitário. J Health Biol Sci. 2018 Jan-Mar; 6(1):54-59. 